\title{
Ecología poblacional de Heteromys pictus (Rodentia: Heteromyidae), en un bosque tropical caducifolio con perturbación humana, en la costa de Oaxaca, México
}

\author{
Miguel Briones-Salas* \& Graciela González \\ Laboratorio de Vertebrados Terrestres (Mastozoología), Centro Interdisciplinario de Investigación para el Desarrollo \\ Integral Regional, Unidad Oaxaca (CIIDIR-OAX.), IPN. Hornos 1003, Santa Cruz Xoxocotlán, Oaxaca, México, CP \\ 71230; mbriones@ipn.mx, gracielago_62@yahoo.com.mx \\ * Correspondencia
}

Recibido 06-VIII-2015. Corregido 22-VI-2016. Aceptado 27-VII-2016.

\begin{abstract}
Population ecology of Heteromys pictus (Rodentia: Heteromyidae), in a tropical deciduous forest with human disturbance, in Oaxaca coast, Mexico. Spiny pocket mice Liomys and Heteromys are forest-dwelling granivorous rodents distributed in seasonally dry forest or thorn scrub along the Pacific lowlands or xeric regions in the Mexican Plateau. We analyzed the population dynamics of the spiny pocket mouse Heteromys pictus, in the coast of Oaxaca, Mexico, in two sites of tropical deciduous forest with different degrees of disturbance: $\mathrm{ZPP}=$ less disturbed site, and $\mathrm{ZMP}=$ more disturbed site. Two plots were established in each area with 66 trap stations; we used the capture-recapture method and trapping for 12 periods for one year. Population density was estimated using the method of Minimum Number of Individuals Known Alive (MNKA). We captured 706 individuals, 290 for the ZPP and 416 for the ZMP. The population density ranged from 28 to 142 ind./ ha for both zones, but it was higher for the ZMP (54 against 142 ind./ha). The population density for both areas increased during the rainy season and coincided with the increased recruitment of adults. Reproductive activity occurred throughout the study period in both areas, but it was higher for the ZMP. The sex ratio in the ZPP was 1:1 while in the ZMP was 1:0.75. In the ZMP we found a higher population density and reproductive activity in females, compared to the ZPP. These results suggest that $H$. pictus prefers the ZMP, indicating that the species is not significantly affected by human disturbance in the studied location. Rev. Biol. Trop. 64 (4): 1415-1429. Epub 2016 December 01.
\end{abstract}

Key words: capture-recapture, disturbance, Liomys, populations, spiny pocket mouse.

El disturbio ecológico en bosques tropicales causado por actividades humanas comola agricultura, la ganadería y urbanización, comúnmente afecta la composición, estructura y procesos de las comunidades animales (García-Estrada, Romero-Almaraz \& SánchezHernández, 2002; Zalapa, Badii, Cervantes, \& Guerrero, 2005; Jaramillo, García-Oliva, \& Martínez-Yrizar, 2010). Estas perturbaciones provocan parches de vegetación con características ambientales diferentes a la original y pueden afectar la densidad poblacional de animales (Adler, 1987), lo que aumenta la inestabilidad de las poblaciones de las especies y la probabilidad de extinción local (Bowers \& Matter 1997; Zalapa et al., 2005). Mientras que algunas especies residentes pueden desaparecer con la perturbación del hábitat, especies tolerantes o exóticas invasoras, pueden llegarpara explotar las áreas abandonadas (Nupp \& Swihart, 1996, 1998).

Los efectos que tiene la perturbación sobre las poblaciones de las diferentes especies están regulados por la condición "especie-específicas"; es decir, cada especie responde de manera particular a los efectos de perturbación (Bolger et al., 1997; Lynam, 1997; Laidlaw, 2000; Sánchez-Hernández, Romero-Almaraz, 
Colín-Martínez, \& García-Estrada, 2001). Algunas son más sensibles, mientras que otras se ven beneficiadas con las modificaciones, mostrando valores altos de abundancias pero poca estabilidad en sus poblaciones (Adler, Arboledo, \&Travi, 1997). Los recursos alimenticios, la disponibilidad de refugios, la depredación, así como la estructura del hábitat y las condiciones ambientales estacionales (precipitación principalmente), son factores que influyen sobre la composición de las comunidades, patrones demográficos y ciclos reproductivos de las poblaciones de pequeños mamíferos (August, 1983; Krebs, 1985; Adler, 1987; O'Connell, 1989).

El bosque tropical caducifolio de la costa de Oaxaca, al sur de México, muestra una elevada transformación, principalmente por actividades antropogénicas como la agricultura, ganadería, incendios y actividades turísticas (Mass et al., 2010; Lira \& Ceballos, 2010). Por otro lado, es considerado como la última superficie de este ecosistemas en la región (Salas-Morales, Saynes-Vásquez, \& Schibli, 2003) y un sitio con una alta diversidad biológica (González-Pérez, Briones-Salas, \& Alfaro, 2004). Se ha registrado un alto número de especies vegetales (1 300 especies) y algunos grupos de animales como aves (400 especies) y mamíferos (121 especies) (Salas-Morales \& Casariego 2010; Navarro, García-Trejo, Peterson, \& Rodríguez-Contreras, 2004; BrionesSalas, Cortés-Marcial, \& Lavariega, 2015).

Dentro de este último grupo, los roedores de la especie Heteromys pictus Thomas, 1893 (Heteromyidae) (antes llamado Liomyspictus; Hafner et al., 2007; Ramírez-Pulido, GonzálezRuíz, Gardner, \& Arroyo-Cabrales, 2014), son abundantes en los bosques tropicales caducifolios (Ceballos \& Miranda, 2000; Briones-Salas, Sánchez-Cordero, \& Sánchez-Rojas, 2006; Ceballos \& Martínez, 2010). Característico de estos animales son las adaptaciones que los hacen exitosos en cuanto a sobrevivencia y reproducción en ambientes austeros, en comparación con otras especies (McGhee \& Genoways, 1978; Ceballos \& Valenzuela, 2010). Por ser una especie removedora de semillas, desempeña un papel importante en la regeneración del bosque (Janzen, 1971; Sánchez-Rojas, Sánchez-Cordero, \& BrionesSalas, 2004; Briones-Salas et al., 2006), y se ha observado que la dinámica poblacional de estos heterómidos tiene una relación directa con los periodos de fructificación de las plantas (Fleming, 1971, Fleming, 1974; Janzen, 1986; Romero, 1993; Briones, 1996).

Algunos autores, han mencionado como las principales causas de la conservación de esta especie y otras de heterómidos, el cambio en el uso del suelo, la destrucción del hábitat y las tendencias poblacionales (Fernández, Hafner, Hafner, \& Cervantes, 2014). Los estudios sobre el efecto que tiene la perturbación del ambiente por las actividades humanas, sobre la dinámica poblacional de $H$. pictus son escasos. Estos estudios han demostrado que la especie no es afectada por la pérdida de cobertura vegetal de manera importante, ya que no modifica su comportamiento, ni la intensidad de su actividad, por lo que resulta una especie tolerante a la perturbación (García-Estrada et al., 2002; Gutiérrez-Ramos \& Álvarez-Castañeda, 1999).

Por esta razón, resulta importante, conocer la dinámica poblacional de $H$. pictus, ya que de esta manera se podrá entender el papel que juegan estos mamíferos en la dinámica del bosque tropical caducifolio, así como sus implicaciones para el conocimiento y conservación de este ecosistema. Por lo tanto, el presente estudio tiene como objetivo conocer la dinámica poblacional del ratón espinoso de abazones (H. pictus), en sitios con diferente grado de perturbación antropogénica en un bosque tropical caducifolio de la región Planicie Costera del Pacífico en el estado de Oaxaca, México.

\section{MATERIALES Y MÉTODOS}

Área de estudio: El estudio se desarrolló en el Municipio de San Miguel del Puerto, en la Planicie costera del Pacífico, Oaxaca, México (Fig. 1). El clima es cálido subhúmedo (Aw), con una estacionalidad marcada, la temporada seca de noviembre a abril y la lluviosa de mayo 


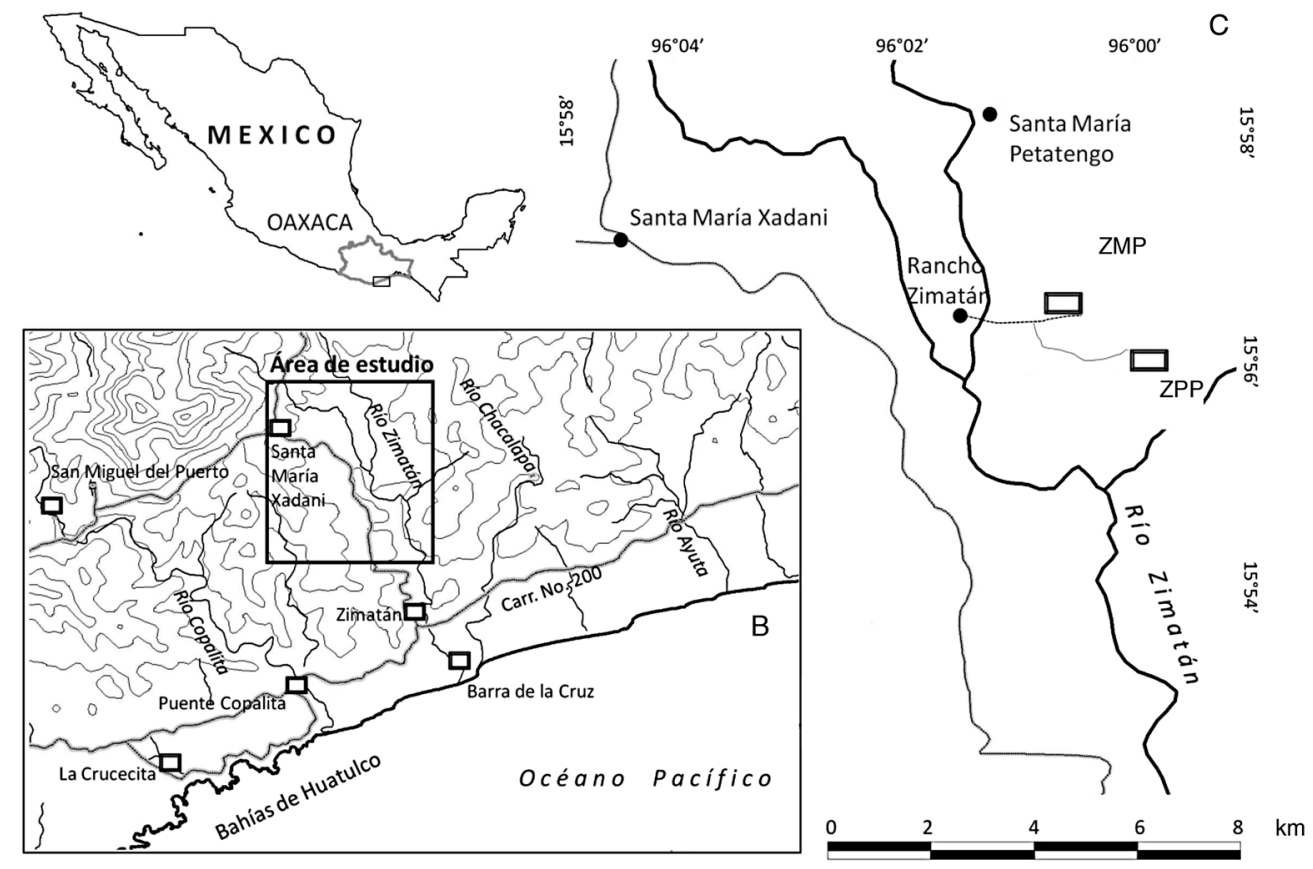

Fig. 1. Zona de estudio. B. Zona de muestreo en la costa de Oaxaca, México. C. Ubicación de los cuadrantes muestreados $(\mathrm{ZPP}=$ zona poco perturbada y $\mathrm{ZMP}=$ zona más perturbada).

Fig. 1. Study area. B. Sampling in the coastal of Oaxaca, Mexico. C. Sampled quadrants location $(Z P P=$ less disturbed area and $\mathrm{ZMP}=$ more disturbed area).

a octubre; la temperatura media anual es de $28.3{ }^{\circ} \mathrm{C}$ (Fig. 2).

La vegetación dominante es bosque tropical caducifolio y bosque tropical subcaducifolio; ambos pierden sus hojas durante la época seca del año y florísticamente son muy similares (Salas-Morales et al., 2003). El bosque tropical caducifolio, se caracteriza por tener una gran variabilidad en su altura, que va de 8 a $25 \mathrm{~m}$. Estos bosques se distinguen por presentar dos estratos arbóreos; el alto con especies de Lonchocarpus aff. magallanesii, Euphorbia

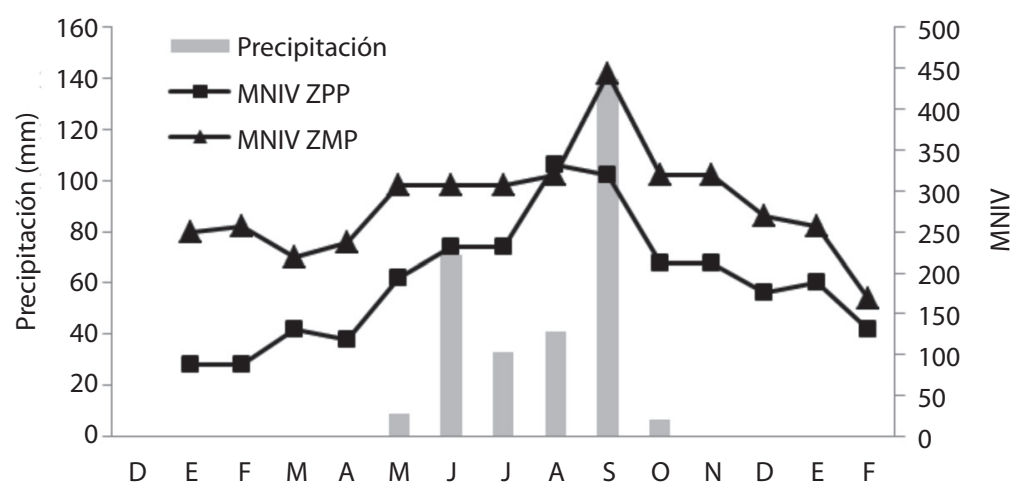

Fig. 2. Precipitación pluvial y fluctuación poblacional de Heteromys pictus en una ZPP y ZMP en un bosque tropical caducifolio de la costa de Oaxaca, México; datos de 2015 obtenidos de la estación meteorológica de Puerto Ángel, Oaxaca. Fig. 2. Rainfall and population fluctuation of Heteromys pictus in a ZPP and ZMP in a tropical deciduous forest on the coast of Oaxaca, Mexico; data obtained of 2005 from the meteorological station of Puerto Angel, Oaxaca. 
calyculata, Gyrocarpus americanus, Cordia sonorae y Cordia tinifolia, e indistintamente en ambos estratos especies como: Albizia occidentalis, Amphipterygium adstringens, Bursera excelsa, Bursera simaruba, Jacaratia mexicana y Pterocarpus acapulcensis, entre otras más (Salas-Morales et al., 2003). Dentro de estos bosques, existen sitios con diferente grado de perturbación antropogénica principalmente por la siembra de maíz de temporal y presencia de ganado (Salas-Morales \& Casariego, 2010).

Se seleccionaron dos sitios separados por $1.5 \mathrm{~km}$, de acuerdo a la perturbación humana y al tiempo de regeneración de la vegetación: sitio 1, bosque tropical caducifolio con aproximadamente 13 años de regeneración, a 271 msnm (155' $\left.40.75^{\prime \prime} \mathrm{N}-96^{\circ} 01^{\prime} 25.33^{\prime \prime} \mathrm{W}\right)$; sitio 2, bosque tropical caducifolio y vegetación secundaria con menos de cinco años de regeneración; éste se encuentra cercano $(<1 \mathrm{~km})$ a sitios de cultivos de maíz y frijol y presenta mayor actividad de ganado, a 240 msnm (1556'23.3" N - 96 $\left.06^{\circ} 1.45^{\prime \prime} \mathrm{W}\right)$.

Muestreo de roedores: En cada sitio de muestreo (2) se establecieron dos cuadrantes, apartados $600 \mathrm{~m}$ entre sí; cada uno con 66 estaciones de trampeo (11 líneas y 6 columnas), con una separación de 10 m cada una, cubriendo una área de 0.50 ha.

En cada estación de muestreo, se colocó una trampa Sherman ${ }^{\mathrm{TM}}(7.5 \times 9.0 \times 23 \mathrm{~cm})$, cebada con hojuelas de avena y vainilla. Se realizaron 12 muestreos de cuatro noches consecutivas por sitio, entre enero 2004 y febrero 2005, se excluyeron los correspondientes a los meses de junio y noviembre 2004, por problemas de logística. Se empleó el método de captura-marcaje-recaptura, por medio de la técnica de ectomización de falanges (Rudran, 1996). Para cada individuo capturado se determinó la especie, peso, sexo y medidas convencionales (largo total, largo cola, largo pata, largo oreja y peso), al igual que la fecha y ubicación de la estación de trampeo. La edad se clasificó en tres categorías: joven $(<190 \mathrm{~mm}$, pelaje poco denso, testículos abdominales en machos y vagina cerrada y/o sin pezones para las hembras); adultos (> $190 \mathrm{~mm}$, pelaje denso o con muda, para machos testículos escrotados y sacos epididimales alargados y para hembras vagina abierta y/o desarrollo mamario grande o lactante); finalmente, subadultos (machos con testículos inguinales o escrotados, sin sacos epididimales evidentes y con pelaje incompleto en las extremidades) (Hernández-Betancourt, López-Wilchis, Cimé, \& Medina, 2003). Se registró la condición reproductiva (activos, cuando los machos presentaron testículos escrotados y sacos epididimales alargados y las hembras vagina abierta, desarrollo mamario grande o lactando o se palparon embriones; e inactivos cuando presentaron lo contrario). Después de registrar los datos, los animales se liberaron en el mismo lugar de su captura. Cuando los ejemplares fueron recapturados se registró, además de lo antes citado, el número asignado a cada animal y la ubicación de la estación de trampeo.

Como reclutas se consideraron individuos que se marcaron nuevos dentro de la zona de manera mensual (Galindo-Leal \& Krebs, 1997). La residencia se determinó considerando la permanencia máxima en meses de un individuo dentro de la zona, desde su primera hasta la última captura.

La densidad poblacional se determinó por el método del Número Mínimo de Individuos Conocidos Vivos (NMIV; Krebs, 1966; Nichols \& Pollock, 1983). Para comprobar el supuesto de que la mayoría de los individuos son capturados, se obtuvo el índice de trampeo (IT; Krebs, Wingate, Leduc, Redfield, Taitt, \& Hilbron, 1976): IT= número de individuos capturados al tiempo i/ número de individuos estimados presentes al tiempo i, (Ni).

El IT obtenido se multiplicó por 100, para obtener el resultado en porcentaje. La diferencia entre los parámetros: densidad poblacional, estructura de edades y actividad reproductiva por zona y por temporada, se analizaron mediante un análisis de varianza (ANOVA), utilizando los dos sitios de estudio y las temporadas (seca y lluviosa) como factores. Las diferencias entre la frecuencia de individuos de cada sexo y de cada temporada para cada 
zona, se analizaron mediante una prueba de $X^{2}$. Se utilizó el coeficiente de correlación de Spearman para medir el grado de asociación entre la densidad poblacional y la precipitación pluvial en ambas zonas (Mendenhall, Wackerly, \& Scheaffer, 1994). Para todos los parámetros se consideran diferencias significativas cuando $\mathrm{P} \leq 0.05$.

Posterior al estudio de los roedores, se analizó el grado de perturbación antropogénica de los dos cuadrantes de cada sitio de muestreo, con los siguientes factores: 1) Heterogeneidad ambiental. Se realizaron dos recorridos en línea en cada cuadrante durante cada temporada (lluviosa y seca) para el conteo de microhábitats (suelo-hojarasca, roca, tronco vivo y tronco muerto). 2) Cobertura del estrato arbóreo. Se estimó el porcentaje promedio de tres puntos al azar dentro de cada cuadrante, obtenidos con un densitómetro esférico. 3) Cobertura del estrato arbustivo y estrato herbáceo. Se estimó el porcentaje promedio de 3 parcelas de $2 \times 2 \mathrm{~m}$ para el estrato arbustivo y 3 parcelas de $1 \times 1$ $\mathrm{m}$ para el herbáceo medido con un flexómetro convencional para cada cuadrante (cm). 4) Pendiente del terreno. Se calculó en un mapa topográfico de la zona a una escala de 1:50000, de acuerdo a las curvas de nivel y se consideraron las categorías: $0-20^{\circ}=$ bajo, $21-40^{\circ}=$ moderado, $41-60^{\circ}=$ pronunciado). 5) Agricultura dentro de los cuadros o a una distancia menor a $15 \mathrm{~m}(0=$ no; 1 = anual, 2 = temporal $) .6)$ Cercanía a poblados $(\mathrm{km})$.

\section{RESULTADOS}

Factores de perturbación: El sitio 1 registró menor perturbación, por lo que se le denominó "zona poco perturbada" (ZPP); se registró mayor cobertura arbórea y arbustiva, se encuentra más alejado a poblados y menor presencia de ganado. La mayor perturbación se registró en el sitio 2, se le denominó "zona más perturbada" (ZMP); presenta mayor cantidad de troncos muertos, menor cobertura arbórea y mayor cobertura herbácea; además, a menos de $100 \mathrm{~m}$ se encontraban zonas de cultivo (Cuadro 1).

Comunidad de roedores: Se registraron 1215 capturas de individuos en ambas zonas, 494 para la ZPP y 721 para la ZMP, que corresponden a seis especies de dos familias (Heteromyidae y Cricetidae). En la ZPP se capturaron Heteromys pictus $(\mathrm{N}=290 ; 58.7 \%)$,

\section{CUADRO 1}

Factores de perturbación (ambientales y actividad humana) en una zona poco perturbada (ZPP) y en una zona con mayor perturbación (ZMP), en el bosque tropical caducifolio de la costa de Oaxaca, México

TABLE 1

Disturbance factors (environmental and human activity) in a less disturbed area (ZPP) and in a more disturbance area (ZMP), in the tropical deciduous forest on the coast of Oaxaca, Mexico

\begin{tabular}{|c|c|c|c|c|}
\hline & ZPP 1 & ZPP 2 & ZMP 1 & ZMP 2 \\
\hline \multicolumn{5}{|l|}{ Heterogeneidad } \\
\hline Suelo-hojarasca (\%) & 45 & 50 & 60 & 65 \\
\hline Roca (número de rocas $>30 \mathrm{~cm}$ ) & 10 & 6 & 8 & 9 \\
\hline Tronco vivo (número de troncos $>10 \mathrm{~cm}$ ) & 27 & 33 & 17 & 22 \\
\hline Tronco muerto (número de troncos $>10 \mathrm{~cm}$ ) & 6 & 7 & 15 & 18 \\
\hline Cobertura del estrato arbóreo (\%) & 80 & 70 & 40 & 30 \\
\hline Cobertura del estrato arbustivo (\%) & 60 & 50 & 35 & 15 \\
\hline Cobertura del estrato herbáceo $(\mathrm{cm})$ & 50.4 & 60.8 & 175.1 & 185.4 \\
\hline Pendiente & $0-20^{\circ}$ & $21-40^{\circ}$ & $21-40^{\circ}$ & $0-20^{\circ}$ \\
\hline Agricultura $(0=$ ausente, $1=$ presente $)$ & 0 & 0 & 1 & 1 \\
\hline Cercanía a poblados $(\mathrm{km})$ & 4 & 4 & 3 & 3 \\
\hline
\end{tabular}


Peromyscus aztecus $(\mathrm{N}=188 ; 38 \%)$ y Oryzomys couesi $(\mathrm{N}=16 ; 3.24 \%)$. Para la ZMP se registraron $H$. pictus $(\mathrm{N}=416 ; 57.70 \%)$, P. aztecus $(\mathrm{N}=236 ; 32.73 \%)$, O. couesi $(\mathrm{N}=$ 41; $5.69 \%)$, Mus musculus ( $\mathrm{N}=74 ; 10.26 \%)$ y Reithrodontomys fulvescens $(\mathrm{N}=2 ; 0.28 \%)$.

Índice de trampeo y permanencia máxima de $\boldsymbol{H}$. pictus: El índice de trampeo en la ZPP fue superior al $80 \%$ durante ocho periodos de muestreo, durante la temporada de lluvia se registró el índice más alto ( $83 \%$, Cuadro 2). En la ZMP se presentaron índices de trampeo superiores a $80 \%$ en seis periodos; durante la temporada seca de 2005 se registró el valor más alto (86\%, Cuadro 3).

La permanencia de los individuos en ambas zonas fue corta, ya que la mayoría de los individuos (> $75 \%$ ) tuvieron residencia de solo un mes. En la ZPP se registraron una hembra y un macho con una permanencia de 13 meses cada uno. En la ZMP se registraron dos hembras con una permanencia de 13 y 14 meses.

Densidad poblacional de $\boldsymbol{H}$. pictus: La densidad poblacional total fluctuó entre 28 y 142 ind./ha $(\overline{\mathrm{X}}=74$ ind./ha; D.E. $=18.38)$. Los picos se presentaron durante la temporada de lluvia en agosto $(\mathrm{N}=106 \mathrm{ind}$./ha) para la ZPP y septiembre (142 ind./ha) para la ZMP, mientras que las densidades más bajas se observaron durante la temporada seca para la ZPP (enero 2004; 28 ind./ha) y febrero 2005 (54 ind./ha) para la ZMP. A pesar de registrarse un valor alto en la correlación entre la densidad poblacional por periodo y la precipitación pluvial mensual en ambas zonas, no resultaron significativas (correlación de Spearman, ZPP, $r_{s}=0.7445, \mathrm{P}=$ 0.0569; ZMP, $\left.r_{s}=0.8597, \mathrm{P}=0.0657\right)$.

En la ZPP la densidad poblacional varió de 28 a 106 ind./ha $(\bar{X}=58.8$; D.E. $=9.9)$, el pico máximo se presentó durante la temporada de lluvia, mientras que los valores más bajos se observaron durante la temporada seca 2004 (Fig. 2; Cuadro 2). Se encontraron diferencias significativas entre ambas temporadas (ANOVA, $\left.\mathrm{F}_{(2,9)}=11.40, \mathrm{P}<0.0034\right)$.
En la ZMP la densidad fluctuó de 54 a 142 ind./ha $(\overline{\mathrm{X}}=67$; D.E. $=18.3)$, el valor máximo se registró durante las lluvias, y el más bajo durante la temporada seca 2005 (Fig. 2; Cuadro $3)$. Se encontraron diferencias significativas entre las temporadas para esta zona (ANOVA, $\left.\mathrm{F}_{(2,9)}=5.61, \mathrm{P}<0.0262\right)$.

La densidad poblacional fue mayor para la ZMP, las pruebas estadísticas mostraron que existen diferencias significativas entre las dos zonas $\left(\mathrm{ANOVA}, \mathrm{F}_{(1,12)}=9.77, \mathrm{P}<0.0049\right.$ ).

Reclutamiento: En la ZPP el mayor y menor número de reclutas se observó durante la temporada de lluvias (agosto, 18 ind. y octubre, 1 ind.). El promedio para ambos sexos fue de 7.85 ind. $(\mathrm{n}=55$; D.E. $=4.22)$ para la temporada seca, $y$ de 10.6 ind. $(n=53$; D.E. $=6.50)$ para la temporada de lluvia (Cuadro 2).

En la ZMP el mayor y menor reclutamiento se observó durante la temporada seca 2004 (enero, 29 ind., y marzo, 7 ind. respectivamente). El reclutamiento promedio para ambos sexos fue de 12.71 ind. ( $=89$; D.E. $=7.65)$ para la temporada seca y 14.4 ind. $(n=72 ;$ D.E. $=6.54)$ para la temporada de lluvia (Cuadro 3).

Proporción de sexos y estructura de edades: En la ZPP, la proporción de sexos fue de 1:1.1 (Cuadro 1), no se observaron diferencias significativas (Chi cuadrada, $\mathrm{X}^{2}=0.882$, g.l. $=1, \mathrm{P}>0.01)$. Durante la temporada seca se registró una proporción de 1:0.95 (hembras $=$ 42 , machos $=40$ ), no se observaron diferencias significativas (Chi cuadrada, $\mathrm{X}^{2}=0.048$, g.l. $=$ $1, \mathrm{P}>0.01)$. Para la temporada de lluvia, la proporción fue de 1:0.94 (hembras $=71$, machos $=$ 70) $\sin$ diferencias significativas (Chi cuadrada, $\mathrm{X}^{2}=0.028$, g.1. $\left.=1, \mathrm{P}>0.01\right)($ Cuadro 2).

Dentro de la ZMP, la proporción de sexos favoreció de manera significativa a las hembras 1: 0.75 (Chi cuadrada, $X^{2}=10.80$, g.l. $=1, \mathrm{P}<0.005)$. En la temporada seca la proporción fue mayor para las hembras 1:0.44 (Chi cuadrada, $\mathrm{X}^{2}=23.72$, g.l. $=1, \mathrm{P}<0.005$ ), mientras que durante la temporada de lluvias la proporción fue de 1:0.9, sin diferencia 


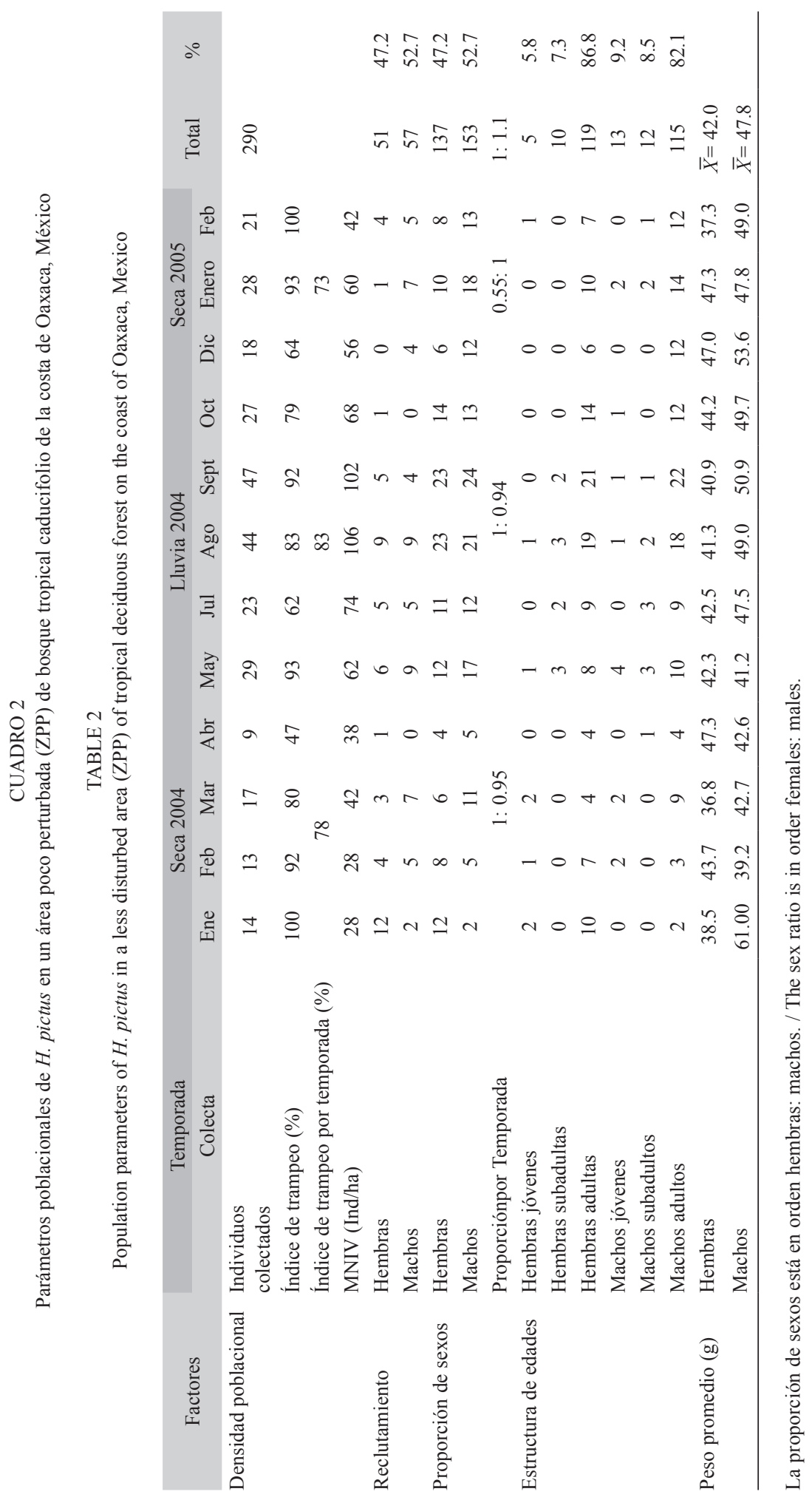




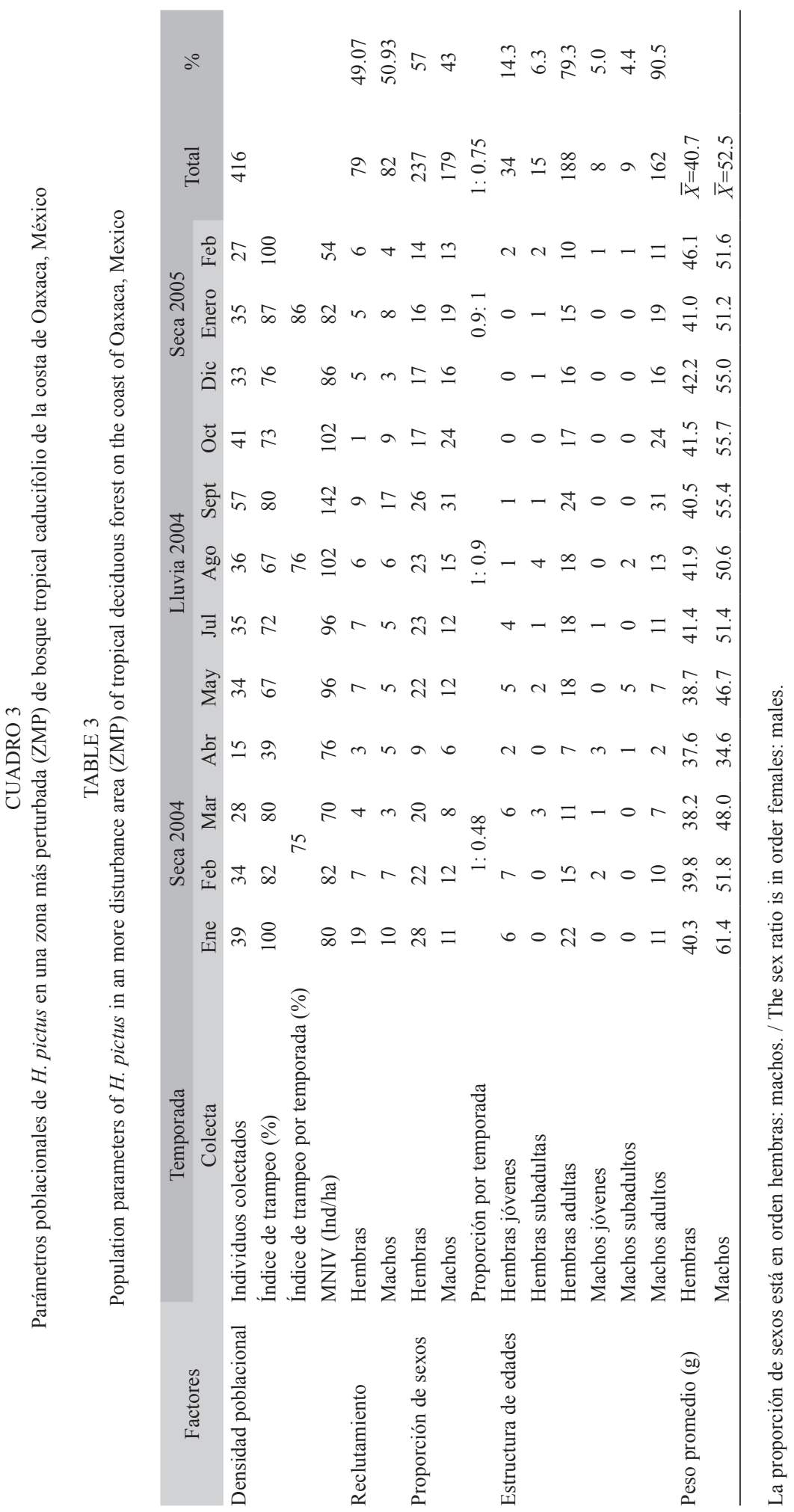


significativa (Chi cuadrada, $\mathrm{X}^{2}=0.28$, g.l. $=1$, $\mathrm{P}>0.01$ ) (Cuadro 3).

En ambas zonas la mayoría de los individuos fueron adultos (> 70\%) (Cuadro 2 y 3 ). En la ZPP, la mayoría de hembras fueron adultas $\left(\mathrm{n}=119 ; 86.8 \%\right.$ ) (ANOVA de dos vías, $\mathrm{F}_{(2,33)}$ $=31.41, \mathrm{P}<0.001)$. El pico se observó durante la temporada de lluvia (septiembre, 21 ind.) y los valores más bajos durante la temporada seca 2004 (marzo, 4 ind.) (Cuadro 2). En los machos, la mayoría fueron adultos $(\mathrm{n}=115$; $82.1 \%$ ) (ANOVA, $\mathrm{F}_{(2,33)}=28.72, \mathrm{P}<0.001$ ). El valor máximo se presentó durante la temporada de lluvia (septiembre, 22 ind.) y el mínimo durante la seca (enero 2004; 2 ind.) (Cuadro 2).

Dentro de la ZMP, se encontró una gran cantidad de hembras adultas $(\mathrm{n}=188 ; 79.3 \%)$ (ANOVA de dos vías, $\mathrm{F}_{(2,33)}=71.42, \mathrm{P}<0.000$ ). El pico se observó en la temporada de lluvia (septiembre, 24 ind.) y el valor más bajo en la seca 2004 (abril, 7 ind.) (Cuadro 3). Para el caso de los machos, los adultos fueron más abundantes $(\mathrm{n}=162 ; 90.5 \%)\left(\right.$ ANOVA, $\mathrm{F}_{(2,33)}=$ 29.35, P 0.001). Los picos se observaron durante la temporada de lluvia (septiembre y octubre, 31 y 24 ind., respectivamente) (Cuadro 3).

Actividad reproductiva: En la ZPP, el $67.8 \%$ de hembras adultas estuvieron inactivas ( $\mathrm{n}=93$ ind.), mientras que el $32.2 \%(\mathrm{n}=44$ ind.) presentaron actividad. Los mayoría de hembras activas se presentaron en la temporada seca (mayo 2004, 7 ind. y enero 2005, 10 ind.) (Fig. 3 A). No se encontraron diferencias significativas entre temporadas (ANOVA, $\mathrm{F}_{(2,9)}$ $=3.92, \mathrm{P}<0.042)$. Los machos activos se presentaron en mayor porcentaje $(\mathrm{n}=81$ ind.; $52.94 \%$ ). La actividad reproductiva se registró durante todo el periodo de estudio (Fig. 3 A), el pico se presentó durante la temporada de lluvias (agosto, 17 ind.), no se observaron diferencias significativas entre temporadas (ANOVA, $\left.\mathrm{F}_{(2,9)}=3.09, \mathrm{P}>0.05\right)$ (Fig. $3 \mathrm{~A}$ ).

Dentro de la ZMP, se registró un mayor porcentaje de hembras adultas inactivas $(62 \%$; $\mathrm{n}=147$ ind.), en comparación con las activas (38 \%; $\mathrm{n}=90$ ind.). Las hembras activas, se encontraron a lo largo de todo el estudio en esta zona, con picos durante la temporada seca 2004, (13 ind.) (Fig. 3 B); sin embargo, no hubo diferencias significativas entre temporadas (ANOVA, $\mathrm{F}_{(2,9)}=1.73, \mathrm{P}>0.5$ ). Por su parte, los machos activos fueron más abundantes en esta zona ( $76 \% ; \mathrm{n}=136$ ind.). El pico se registró durante la temporada de lluvia (septiembre, 28 ind.). La actividad reproductiva fue más alta durante la temporada de lluvia con respecto a la temporada seca (ANOVA, $\mathrm{F}_{(2,9)}=$ 6.31, $\mathrm{P}<0.0194)$.

Finalmente, se observó una mayor cantidad de hembras activas en la ZMP (ANOVA, $\left.\mathrm{F}_{(1,22)}=8.16, \mathrm{P}<0.01\right)$. Para los machos no se encontraron diferencias significativas en la

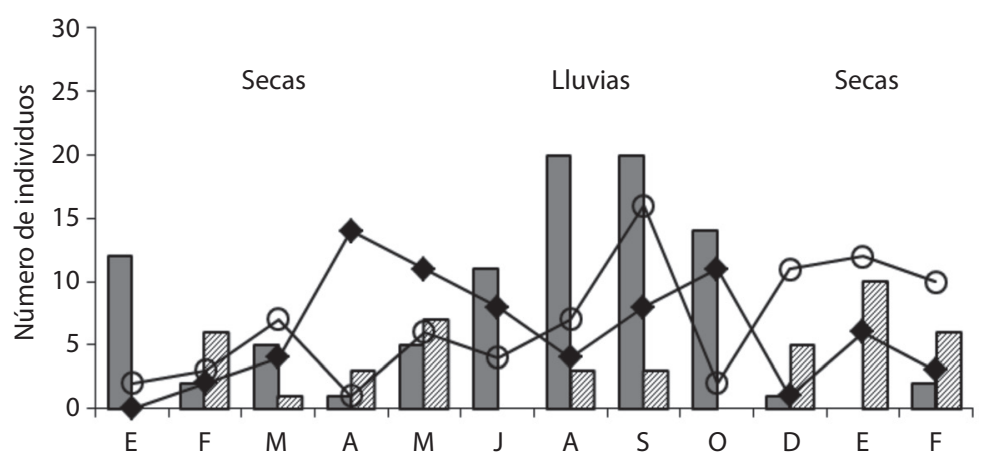

Fig. 3. Actividad reproductiva de Heteromys pictus en una ZPP (A) y una ZMP (B) de bosque tropical caducifolio de la costa de Oaxaca, México.

Fig. 3. Reproductive activity of Heteromys pictus in a ZPP (A) and ZMP (B) of tropical deciduous forest of the coast of Oaxaca, Mexico. 
cantidad de machos activos entre ambas zonas $\left(\right.$ ANOVA, $\left.\mathrm{F}_{(1,22)}=2.88, \mathrm{P}>0.05\right)$.

\section{DISCUSIÓN}

La densidad poblacional de $H$. pictus en ambas zonas varió entre 28 a 142 ind./ha con un promedio mensual de 74 ind./ha. Estos resultados, se encuentran dentro de lo reportado para otros sitios como el bosque tropical caducifolio en Chamela, Jalisco y la Sierra de Huautla, Morelos, ambas en México, con densidades entre 2 y 191 ind./ha (López-Forment, Sánchez-Hernández, \& Villa-Ramírez, 1971; Collett, Sánchez-H., Shum Jr., Teska, \& Baker, 1975; Briones, 1996; García-Estrada et al., 2002).

La densidad poblacional de $H$. pictus, en este estudio, sigue un patrón similar en ambas zonas, empieza a aumentar al inicio de la temporada de lluvia y alcanza los valores máximos al final de la temporada de lluvias, para después disminuir en la siguiente temporada seca. Patrones similares fueron reportados por García-Estrada et al. (2002) en un bosque tropical caducifolio con perturbación humana en Morelos, México, y por Zalapa et al. (2005) en un bosque tropical subcaducifolio de Jalisco, México. Sin embargo, en sitios más conservados como en Chamela, Jalisco, la mayor densidad poblacional se registró durante la temporada seca (Briones, 1996).

La mayor densidad poblacional se encontró en la ZMP, posiblemente como consecuencia de que en esta zona existe una mayor cobertura del estrato arbustivo de sucesión secundaria. En el caso de bosques tropicales que han sido talados o quemados, se ha visto que la productividad primaria puede ser alta debido a los nutrientes, luz y disponibilidad de agua dentro del área (Canham \& Marks, 1985; Maass, 1995), por lo que la abundancia de los pequeños mamíferos dentro de la comunidad, tiene una relación con los cambios en el hábitat (Álvarez \& Arroyo-Cabrales, 1990; Utrera et al., 2000).

Heteromys pictus se ha registrado en áreas perturbadas de bosques tropicales caducifolios y templados, así como en zonas cercanas a cultivos (Vázquez, Medellín, \& Cameron, 2000; García-Estrada et al., 2002). Esta especie incluye una variedad considerable de semillas dentro de su dieta, por lo que tiene alternativas para su alimentación en caso de que falte alguna especie en particular; además, puede tolerar cambios en el ambiente en que vive, en comparación con otras especies que son más especialistas en su alimentación (Briones-Salas \& Sánchez-Cordero, 1999; Domínguez, 2000).

Por otro lado, el número alto de la población de este roedor en sitios perturbados ayuda a la regeneración de las selvas. El doble papel que tienen los individuos de esta especie dentro de la estructura vegetal como agente dispersor y depredador de semillas, es de gran importancia para aquellas especies vegetales de las cuales se alimenta (Ceballos, 1990; Domínguez, 2000; Zalapa et al., 2005).

En este estudio se reporta una baja proporción de individuos que alcanzan el año de permanencia, tres en la ZPP y ocho en la ZMP. La corta residencia de $H$. pictus en ambas zonas, indica que hay una alta proporción de individuos que entran y salen de los sitios; estos movimientos pueden ser debido a que los individuos solo transitan por el área, y emigran en busca de un nuevo territorio, lo que posiblemente ayude a la dispersión de las semillas producidas en el sitio perturbado. En otros estudios también se han reportado tiempos de sobrevivencia reducidos. Fleming (1971), reporta en Panamá que el $25 \%$ de la población de Liomys adspersus sobrevive hasta un año. En Chamela, Jalisco y en Morelos, en México, hembras de $H$. pictus, alcanzan intervalos de dos a 17 meses de permanencia (Briones, 1996; García-Estrada et al., 2002).

En ambas áreas se observaron proporciones altas de reclutas adultos al final de la temporada seca y durante la temporada lluviosa. El aumento de reclutas en la temporada de lluvias coincide con el incremento en la densidad poblacional en ambas zonas, pero no se ve reflejada en un aumento en la cantidad de hembras con actividad sexual como en otros estudios, donde se correlacionan estos factores 
(Romero, 1993; Briones, 1996). Sin embargo, en los machos si se presenta, para ambas zonas, una relación de densidad poblacional, reclutamiento y actividad reproductiva durante la temporada lluviosa. Probablemente los machos ingresan en mayor cantidad para aparearse con las hembras locales y por tanto las hembras no tienen que trasladarse hacia otra área para esta función (Diffendorfer, Gaines, \& Holt, 1995).

La proporción de sexos final en la ZPP fue de 1:1, se aprecia un cambio en la proporción durante la época seca favoreciendo a los machos, temporada en la cual hay un bajo reclutamiento de hembras en la zona. En la ZMP la proporción de sexos está sesgada hacia las hembras durante la temporada seca. Romero (1993) indica que la proporción de sexos para $H$. pictus al nacimiento podría ser de 1:1 y después intervienen otros factores que sesgan la población, como la dispersión de sexos, depredación o el temor a entrar a las trampas (trampofobia).

Otro factor que intervino en la proporción de sexos, fue la estancia de los organismos dentro de los cuadrantes; en la ZMP hay una permanencia más prolongada de hembras, que junto con un mayor reclutamiento contribuye a que haya una mayor presencia de hembras. A través de diferentes estudios se ha visto que la proporción de sexos difiere, se ha reportado una proporción de 1:1 (Briones, 1996) en Chamela, Jal., o sesgada hacia las hembras en Jalisco y Morelos, México (Romero, 1993; Vázquez et al., 2000; Zalapa et al., 2005). En la zona del canal de Panamá, fue de 1:1 para $H$. salvini, $H$. desmarestianus y $L$. adspersus (Fleming, 1970, 1971, 1974). Estas diferencias entre sitios, muestran que hay variaciones según los hábitats particulares y los tiempos en que se realizan los estudios, ya que en una misma zona geográfica como en Jalisco, México, se observaron variaciones en diferentes años (Romero, 1993; Briones, 1996).

La población de $H$. pictus estuvo compuesta en su mayoría de adultos en las dos zonas de muestreo. Esto coincide con lo obtenido en Jalisco y Morelos en México con especies de Heteromys (Romero, 1993; Briones, 1996;
García-Estrada et al., 2002), y con otros heterómidos en Costa Rica (Fleming, 1974) y en Veracruz, México (Sánchez-Cordero, 1993). La menor cantidad de jóvenes y subadultos dentro de la población, probablemente se deba a que los jóvenes no salen de sus nidos hasta después de cierto tiempo, o bien porque éstos alcanzan un crecimiento rápido (McGhee \& Genoways, 1978).

Durante todo el estudio se observaron individuos activos sexualmente. Para las hembras, fue continua en la ZMP y discontinua en la ZPP. Los machos activos también se observaron durante todo el estudio en ambas zonas de muestreo. En estudios previos, se han reportado diferencias en las épocas de reproducción de H. pictus; Ceballos (1990), Romero (1993) y Briones (1996) en Jalisco, registraron actividad reproductiva en las temporadas lluviosa y seca, pero con mayor concentración durante la seca. En Panamá y Costa Rica se ha reportado hasta un $100 \%$ de los individuos en condición reproductiva durante la época seca, lo que se atribuye principalmente a los periodos de mayor concentración de semillas (Fleming, 1970, 1971, 1974).

En diversos estudios se ha comprobado la relación entre la actividad reproductiva y la disponibilidad de alimento (Fleming, 1970, 1971, 1974; Ceballos, 1990; Briones, 1996). Cuando hay abundancia de recurso, las hembras presentan a más temprana edad el primer periodo reproductivo, y periodos de nacimientos anticipados (Fleming, 1970, 1971, 1974; O'Connell, 1989).

Aunque no se midió la disponibilidad de alimento en este estudio, es posible que la ZMP, presente una mayor cantidad de semillas, dado que la estrategia de las plantas en sitios en sucesión secundaria es la producción de una gran cantidad de semillas (Finegan, 1984; Williams-Linera, Alvarez-Aquino, HernándezAscención, \& Toledo, 2011). La presencia de mayor cantidad de alimento ayuda a sostener los altos niveles energéticos de la crianza y del periodo de lactancia y concluir exitosamente el ciclo reproductivo (Randolph, Randolph, Mattingly, \& Foster, 1977; Millar, 1979). 
Con base en los resultados obtenidos en este estudio, la perturbación antrópica a la que se encuentra sujeta la población de $H$. pictus, aparentemente no tiene un efecto negativo en la estructura poblacional ni en la dinámica poblacional de la especie. Estos resultados permiten inferir que las actividades desarrolladas en esta región se pueden considerar de bajo impacto, al menos para esta especie. El mismo patrón se ha observado para heterómidos en otros sitios de México, con ambientes perturbados por pastoreo en Baja California (Ortega-Rubio et al., 1993; Trujano-Alvarez et al., 2008) y en zonas con vegetación secundaria en Morelos (GarcíaEstrada et al., 2002).

Estudios de este tipo son importantes debido al doble papel que tiene $H$. pictus para la dinámica del bosque como agente dispersor y depredador de un gran número de semillas. El aumento en la población de este roedor puede llegar a beneficiar a la estructura vegetal del bosque tropical caducifolio; sin embargo, si la densidad continúa en aumento, podría llegar a causar desequilibrios en la dinámica poblacional de la vegetación por depredación excesiva de semillas.

\section{AGRADECIMIENTOS}

A las autoridades del Municipio de San Miguel del Puerto, Oaxaca. A R. Ricardez y A. López por permitirnos trabajar en sus terrenos. Al Fondo Mundial para la Naturaleza (WWF) (S069 y QQ82), el Sistema de Investigación Benito Juárez (SIBEJ) (20000506024) y la Coordinación General de Estudios de Posgrado e Investigación), por el apoyo económico. G. Pérez, G. Magaña, A. Trujano, M. Lavariega y B. Riveros realizaron importantes observaciones. MB-S agradece a la Comisión de Operación y Fomento a las actividades Académicas (COFFA) y al programa de Estímulos al Desempeño a la Investigación (EDI), del Instituto Politécnico Nacional, así como al Sistema Nacional de Investigadores (SNI) por su reconocimiento y apoyo.

\section{RESUMEN}

Los ratones espinosos de abazones Liomys y Heteromys son roedores granívoros que se distribuyen en bosques tropicales secos y en matorral espinoso a lo largo de tierras bajas del Pacífico y en regiones xerófilas en la meseta de México. Analizamos la dinámica poblacional del ratón espinoso de abazones Heteromys pictus en la costa de Oaxaca, México, en dos sitios de bosque tropical caducifolio con diferente grado de perturbación humana: $\mathrm{ZPP}=$ zona poco perturbada, y ZMP = zona con mayor perturbación. Se establecieron dos cuadros en cada área con 66 estaciones de trampeo; se utilizó el método de captura-recaptura durante 12 periodos de muestreo. La densidad poblacional se estimó utilizando el método del Número Mínimo de Individuos Conocidos Vivos (NMIV). Se capturaron 706 individuos, 290 para la ZPP y 416 para la ZMP. La densidad poblacional fluctuó entre 28 y 142 ind./ha para ambas zonas; sin embargo esta fue mayor para la ZMP (54 a 142 ind./ha). La densidad poblacional de ambas zonas aumentó durante la temporada de lluvia y coincidió con el mayor reclutamiento. Se registró actividad reproductiva a lo largo de todo el estudio en ambas zonas, pero fue mayor para la ZMP. La proporción de sexos fue de 1:1 en la ZPP, mientras que en la ZMP fue de 1: 0.75 . En la ZMP encontramos una mayor densidad poblacional y actividad reproductiva en las hembras. Estos resultados sugieren que $H$. pictus prefiere la ZMP, lo que indica que en este lugar la especie no es afectada significativamente por la perturbación humana.

Palabras clave: captura-recaptura, Liomys, perturbación, poblaciones, ratón espinoso de abazones.

\section{REFERENCIAS}

Adler, G. (1987). Influence of habitat structure on demography of two rodent species in Easter Massachusetts. Canadian Journal of Zoology, 65, 903-912.

Adler, G., Arboledo, J., \&Travi, B. (1997). Diversity and abundance of small mammals in degraded tropical dry forest of northern Colombia. Mammalia, 61, 361-370.

Álvarez, T., \& Arroyo-Cabrales, J. (1990). Cálculos de captura, densidad y ámbito hogareño de tres especies de roedores en un área de influencia humana en el sureste de Durango, México. Anales de la Escuela Nacional de Ciencias Biológicas, 33, 185-210.

August, P. (1983). The role of habitat complexity and heterogeneity in structuring tropical mammal communities. Ecology, 64, 1495-1507.

Bolger, D., Alberts, C., Sauvajot, R., Potenza, D., Mccalvin, L., Tran, D., Mazzoni, S., \& Soule, M. (1997). Responses of rodents to habitat fragmentation in 
Coastal Southern California. Ecological Application, $7,552-563$

Bowers, M., \& Matter, S. (1997). Landscape Ecology of Mammals: Relationships between Density and Patch Size. Journal of Mammalogy, 78, 999-1013.

Briones, M. (1996). Estudio sobre la remoción postdispersión de frutos y semillas por mamíferos de un bosque tropical caducifolio (Tesis Doctoral). Universidad Nacional Autónoma de México, México, D.F.

Briones-Salas, M., Sánchez-Cordero, V., \& Sánchez-Rojas, G. (2006). Multi-species fruit and seed removal in a tropical deciduous forest in Mexico. Canadian Journal of Botany, 84, 433-442.

Briones-Salas, M., Cortés-Marcial, M., \& Lavariega, M. (2015). Diversidad y distribución geográfica de los mamíferos terrestres del estado de Oaxaca, México. Revista Mexicana de Biodiversidad, 86, 685-710.

Canham, C., \& Marks, P. (1985). The response of woody plants to disturbance: patterns of establishment and growth. En T. A. Pockett \& P. S. White (Eds.), The ecology of natural disturbance and patch dynamics (pp. 197-216). USA: Academic Press Inc.

Ceballos, G. (1990). Comparative natural history of small mammals from tropical forests in western Mexico. Journal of Mammalogy, 71, 263-266.

Ceballos, G. \& Martínez, L. (2010). Mamíferos. En G. Ceballos, L. Martínez, A. García, E. Espinoza, J. Bezaury, \& R. Dirzo (Eds.), Diversidad, amenazas y áreas prioritarias para la conservación de las selvas secas del Pacífico de México (pp. 119-144). México: CONABIO-Fondo de Cultura Económica.

Ceballos, G. \& Miranda, A. (2000). Guía de campo de los mamíferos de la costa de Jalisco. México: Fundación Ecológica de Cuixmala-UNAM.

Ceballos, G. \& Valenzuela, D. (2010). Diversidad, ecología y conservación de los vertebrados de Latinoamérica. En G. Ceballos, L. Martínez, A. García, E. Espinoza, J. Bezaury, \& R. Dirzo (Eds.), Diversidad, amenazas y áreas prioritarias para la conservación de las selvas secas del Pacífico de México (pp. 93-118). México: CONABIO-Fondo de Cultura Económica.

Collett, S., Sánchez-H. C, Shum Jr., K., Teska, W., \& Baker, R. (1975). Algunas características poblacionales demográficas de pequeños mamíferos en dos hábitats mexicanos. Anales del Instituto de Biología, Serie Zoología, 1, 101-123.

Diffendorfer, J., Gaines, M., \& Holt, R. (1995). Habitat fragmentation and movements of three small mammals (Sigmodon, Microtus and Peromyscus). Ecology, 76, 827-839.

Domínguez, Y. (2000). Estructura y contenido de las madrigueras de Liomys pictus en la selva mediana subperennifolia, en la estación de Biología Chamela, Jalisco (Tesis de Licenciatura). Universidad Nacional Autónoma de México, México, D.F.

Fernández, J., Hafner, M., Hafner., D., \& Cervantes, F. (2014). Conservation status of rodents of the families Geomyidae and Heteromyidae of Mexico. Revista Mexicana de Biodiversidad, 85,576-588.

Finegan, B. (1984). Forest succesion. Nature, 312, 109-114.

Fleming, T. H. (1970). Notes on the rodent faunas of two Panamanian forests. Journal of Mammalogy, 51, 473-490.

Fleming, T. H. (1971). Population ecology of three species of neotropical rodents. Miscellaneous Publications, Museum of Zoology University of Michigan, $143,1-77$.

Fleming, T. H. (1974). The population ecology of two species of Costa Rican heteromid rodent. Ecology, $55,493-561$.

Galindo-Leal, C., \& Krebs, C. (1997). Habitat structure and demography variability of habitat specialist: The rock mouse (Peromyscus difficilis). Revista Mexicana de Mastozoología, 2, 72-89.

García-Estrada, C., Romero-Almaraz, M. L., \& SánchezHernández, C. (2002). Comparison of rodent communities in sites with different degrees of disturbance in deciduous forest of southeastern Morelos, Mexico. Acta Zoológica Mexicana (Nueva serie), 85, 153-168.

González-Pérez, G. E., Briones-Salas, M., \& Alfaro, A. (2004). Integración del conocimiento faunístico del estado. En A. J. García-Mendoza, M. J. Ordóñez, \& M. Briones-Salas (Eds.), Biodiversidad de Oaxaca (pp. 349-366). México: Instituto de Biología, Universidad Nacional Autónoma de México-Fondo Oaxaqueño para la Conservación de la Naturaleza-WorldWildlifeFound.

Gutiérrez-Ramos, A., \& Álvarez-Castañeda, S. T. (1999). Seed removal by heteromyd rodents in three habitats of tropical Mexico. En P. F. Folliott, \& A. OrtegaRubio (Eds.), Ecology and management of forests, woodlands, and shrub lands in the dryland regions of the United States and Mexico; perspectives for the 21st Century (pp. 211-222). Arizona: University of Arizona- Centro de Investigaciones Biológicas del Noroeste, S. C. USDA ForestService.

Hafner, M., Light, J., Hafner, D., Hafner, M., Reddington, E., Rogers, D., \& Riddle, B. (2007). Basal clades and molecular systematic of heteromyid rodents. Journal of Mammalogy, 88, 1129-1145.

Hernández-Betancourt, S., López-Wilchis, R., Cimé, J., \& Medina, S. (2003). Área de actividad, movimiento y organización social de Heteromys gaumeri Allen y Chapman, 1897 (Rodentia: Heteromyidae) en una 
selva mediana subcaducifolia de Yucatán, México. Acta Zoológica Mexicana (Nueva serie), 90, 77-91.

Janzen, D. (1971). Seed predation by animals. Annual Review of Ecology and Systematics, 2, 265-492.

Janzen, D. (1986). Mice, big mammals and seed: it matters who defecates what where. En A. Estrada \& T. H. Fleming (Eds.), Frugivores and seed dispersal (pp.251-271). The Netherlands: Dr. W. Junk Publishers, Dordrecht.

Jaramillo, V., García-Oliva, F., \& Martínez-Yrizar, A (2010). La selva seca y las perturbaciones antrópicas en un contexto funcional. En G. Ceballos, L. Martínez, A. García, E. Espinoza, J. Bezaury, \& R. Dirzo (Eds.), Diversidad, amenazas y áreas prioritarias para la conservación de las selvas secas del Pacifico de México (pp. 527-532). México: CONABIO-Fondo de Cultura Económica.

Krebs, C. (1966). Demographic changes in fluctuating populations of Microtus californicus. Ecological Monographs, 36, 239-273.

Krebs, C. (1985). Estudio de la distribución y la abundancia. España: Editorial Harla.

Krebs, C., Wingate, I., Leduc, J., Redfield, J., Taitt, M., \& Hilbron, R. (1976). Microtus population biology: dispersal in fluctuating populations of $M$. townsendii. Canadian Journal of Zoology, 54, 79-95.

Laidlaw, R. K. (2000). Effects of habitat disturbance and Protected Areas on mammals of Peninsular Malaysia. Conservation Biology, 14, 1639-1648.

Lira, I., \& Ceballos, G. (2010). Huatulco, Oaxaca. En G. Ceballos, L. Martínez, A. García, E. Espinoza, J. Bezaury, \& R. Dirzo (Eds.), Diversidad, amenazas y áreas prioritarias para la conservación de las selvas secas del Pacífico de México (pp. 521-526). México: CONABIO-Fondo de Cultura Económica.

López-Forment, W., Sánchez-Hernández, C., \& VillaRamírez, B. (1971). Algunos mamíferos de la región de Chamela, Jalisco, México. Anales del Instituto de Biología, Serie Zoología, 42, 99-106.

Lynam, A. (1997). Rapid decline of small mammal diversity in Monsoon Evergreen Forest fragments in Thailand. En W. F. Laurance \& R. O. Bierregaard (Eds.), Tropical Forests Remnants. Ecology management and Conservation of fragmented communities (pp. 222-239). Chicago: The University Chicago Press.

Maass, J. M. (1995). Tropical deciduous forest conversion to pasture and agriculture. In S. H. Bullock, Mooney, H., \& Medina, E. (Eds.), Seasonally dry tropical forest (pp. 399-422). Cambridge: Cambridge University Press.

Maass, M., Búrquez, A., Trejo, I., Valenzuela, D., González, M., Rodríguez, M., \& Arias, H. (2010). Amenazas.
En G. Ceballos, L. Martínez, A. García, E. Espinoza, J. Bezaury, \& R. Dirzo (Eds.), Diversidad, amenazas y áreas prioritarias para la conservación de las selvas secas del Pacífico de México (pp. 321-346). México: CONABIO-Fondo de Cultura Económica.

Macghee, M., \& Genoways, H. (1978). Liomys pictus. Mammalian Species, 40, 1-6.

Mendenhall, W., Wackerly, D., \& Scheaffer, R. (1994). Estadística matemática con aplicaciones. México. México: Grupo Editorial Iberoamérica.

Millar, J. (1979). Energetics of lactation in Peromyscus maniculatus. Canadian Journal of Zoology, 57, 1015-1019.

Navarro, A., García-Trejo, E., Peterson, T., \& RodríguezContreras, V. (2004). Aves. En A. J. García-Mendoza, M. J. Ordóñez, \& M. Briones-Salas (Eds.), Biodiversidad de Oaxaca (pp. 391-421). México: Instituto de Biología, Universidad Nacional Autónoma de México-Fondo Oaxaqueño para la Conservación de la Naturaleza-WorldWildlifeFound.

Nichols, J., \& Pollock, K. (1983). Estimation methodology in contemporary small mammal capture-recapture studies. Journal of Mammalogy, 64, 253-260.

Nupp, T., \& Swihart, R. (1996). Effect of forest pach area population attributes of white-footed mice Peromyscus leucopus in fragmented landscapes. Canadian Journal of Zoology, 74, 467-472.

Nupp, T., \& Swihart, R. (1998). Effects of forest fragmentation on population attributes of white-footed mice and easter chipmunks. Journal of Mammalogy, $79,1234-1243$

O'Connell, M. (1989). Population dynamics of neotropical small mammals in seasonal habitats. Journal of Mammalogy, 70, 532-548.

Ortega-Rubio, A., Romero-Schmidt, H., Argüelles-Méndez, C., Coria-Benet, R., \& Solis-Marin, F. (1993). Livestock exclusión: consequences on nocturnal rodents in Baja California Sur. Revista de Biología Tropical, 41(3), 907-909.

Ramírez-Pulido, J., González-Ruíz, N., Gardner, A., \& Arroyo-Cabrales, J. (2014). List of Recent Land Mammals of Mexico, 2014. Special Publications of the Museum of Texas Tech University, 63, 1-69.

Randolph, P., Randolph, C., Mattingly, K., \& Foster, M. (1977). Energy costs of reproduction in the cotton rat (Sigmodon hispidus). Ecology, 58, 31-45.

Romero, M. L. (1993). Biología de Liomyspictus (Tesis doctoral). Universidad Nacional Autónoma de México, México, D.F.

Rudran, R. (1996). Methods for marking mammals. In D. E. Wilson, F. R. Cole, J. D. Nichols, R. Rudran, 
\& M. S. Foster (Eds.), Measuring and Monitoring Biological Diversity: Standard Methods for Mammals (pp. 299-310). Washington, D.C.: Smithsonian Institution Press.

Salas-Morales, S., Saynes-Vásquez, A., \& Schibli, L. (2003). Flora de la costa de Oaxaca, México: lista florística de la región de Zimatán. Boletín de la Sociedad Botánica Mexicana, 72, 21-58.

Salas-Morales, S., \& Casariego, M. A. (2010). Zimatán, Oaxaca. En G. Ceballos, L. Martínez, A. García, E. Espinoza, J. Bezaury, \& R. Dirzo (Eds.), Diversidad, amenazas y áreas prioritarias para la conservación de las selvas secas del Pacífico de México (pp. 527-532) México: CONABIO-Fondo de Cultura Económica.

Sánchez-Cordero, V. (1993). Estudio poblacional de la rata espinosa Heteromys desmarestianus en una selva húmeda en Veracruz, México. En G. Ceballos \& R. Medellín (Eds.), Avances en el estudio de los mamíferos de México (pp. 301-316), México: Publicaciones especiales, Vol. I. Asociación Mexicana de Mastozoología, A. C.- Universidad Nacional Autónoma de México.

Sánchez-Hernández, C., Romero-Almaraz, M. L., ColínMartínez, H., \& García-Estrada, C. (2001). Mamíferos de cuatro áreas con diferentes grados de alteración en el sureste de México. Acta Zoológica Mexicana (Nueva Serie), 84, 35-48.

Sánchez-Rojas, G., Sánchez-Cordero, V., \& Briones-Salas, M. (2004). Effect of plant species, fruit density and habitat on post-dispersal fruit and seed removal by spiny pocket mice (Liomys pictus, Heteromyidae) in a tropical dry forest in México. Studies on Neotropical Fauna and Environment, 39, 1-6.

Trujano-Alvarez, A., Santillan-Ortíz, H., \& Alvarez-Castañeda, S. 2008. Efecto del pastoreo en el área de actividad y uso del hábitat de una comunidad de heterómidos en el matorral sarcocaule de Baja California Sur. En C. Lorenzo, E. Espinoza, \& J. Ortega (Eds.), Avances en el estudio de los mamíferos de México (pp. 301-316), México: Publicaciones especiales, Vol. II. Asociación Mexicana de Mastozoología, A. C., México, D.F.

Utrera, A., Duno, G., Ellis, B., Salas, R., de Manzione, N., Fulhorst, C., Tesh, R., \& Mills, J. (2000). Small mammals in agricultural areas of western llanos of Venezuela: community structure, habitat associations and relative densities. Journal of Mammalogy, $81,536-548$

Vázquez, L., Medellín, R., \& Cameron, G. (2000). Population and community ecology of small rodents in montane forest of western Mexico. Journal of Mammalogy, 8, 77-85.

Williams-Linera, G., Álvarez-Aquino, C., HernándezAscención, E., \& Toledo, M. (2011). Early successional sites and the recovery of vegetation structure and tree species of the tropical dry forest in Veracruz, Mexico. New Forest, 42, 131-148.

Zalapa, S., Badii, M., Cervantes, F. A., \& Guerrero, S. (2005). Ecología poblacional de Liomys pictus en tres áreas de bosque tropical subcaducifolio con diferente tiempo de regeneración, en la costa norte de Jalisco, México. Acta Zoológica Mexicana (Nueva Serie), 21, 1-14. 
